\title{
Feynman graph integrals and almost modular forms
}

\author{
Si LI
}

\begin{abstract}
We introduce a type of graph integrals on elliptic curves from the heat kernel. We show that such graph integrals have modular properties under the modular group $S L(2, \mathbb{Z})$, and prove the polynomial nature of the anti-holomorphic dependence.
\end{abstract}

\section{Introduction}

Modular forms arise naturally in physics as correlation functions of quantum system with modular groups as symmetries. One such example is the topological string theory on Calabi-Yau manifolds. The topological string produces geometric invariants $F_{g}$ for each non-negative integer $g$, which can be viewed as generalized modular forms on the Calabi-Yau moduli space. However, $F_{g}$ 's are in general not holomorphic objects. They satisfy the holomorphic anomaly equation as shown in the work of Bershadsky, Cecotti, Ooguri and Vafa (BCOV) [2]. When the Calabi-Yau is an elliptic curve, Dijkgraaf [5] anticipated the interpretation of $F_{g}$ in the language of almost modular forms. Later, Aganagic et al. [1] generalize almost modular forms to describe local Calabi-Yau models in topological strings.

In this paper, we will focus on elliptic curves and their moduli. By "almost modular form" of weight $k$, we mean in a weaker sense than [7]: a function $f(\tau, \bar{\tau})$ on the upper half-plane $\mathcal{H}$, which is modular of weight $k$

$$
f(\gamma \tau, \overline{\gamma \tau})=(C \tau+D)^{k} f(\tau, \bar{\tau}), \quad \forall \gamma=\left(\begin{array}{ll}
A & B \\
C & D
\end{array}\right) \in S L(2, \mathbb{Z}),
$$

where $\gamma \tau=\frac{A \tau+B}{C \tau+D}$, and the anti-holomorphic dependence of $f$ is of polynomial in $\frac{1}{\operatorname{Im} \tau}$, i.e.,

$$
f(\tau, \bar{\tau})=\sum_{i=0}^{N} f_{i}(\tau) \frac{1}{(\operatorname{Im} \tau)^{i}}
$$

for some non-negative integer $N$ and holomorphic functions $f_{i}(\tau)$. The famous $\bar{\tau} \rightarrow \infty$ limit [2] picks up the leading holomorphic term $f_{0}(\tau)$ in this context, which is quasi-modular [7]. 
The current paper is motivated from the recent establishment of higher genus mirror symmetry on elliptic curves $[3,4,8]$. The corresponding topological string invariants have been constructed from the quantization of Kodaira-Spencer theory. We are lead to consider the following integral $W_{\Gamma}$ associated with any graph $\Gamma$ : for each edge of $\Gamma$, we associate a kernel function constructed from the heat kernel; for each vertex, we associate a copy of integration on the elliptic curve. See Section 3 for precise definitions. $W_{\Gamma}$ depends on the complex structure of the elliptic curve, and can be viewed as a function on the upper half-plane.

Theorem 1.1. $W_{\Gamma}$ is an almost modular form of weight $2|E(\Gamma)|$ in the above sense. Here $|E(\Gamma)|$ is the number of edges in $\Gamma$.

We can also put certain holomorphic derivatives on the propagator and obtain the graph integral for a decorated graph. The resulting graph integral is again almost modular form with specific weight. The precise statement is given in Proposition 5.2, and Theorem 1.1 follows as a special case. As shown in $[4,5,3,8]$, the topological string invariants on elliptic curves are given by combinatorial sum of graph integrals of this type.

The paper is organized as follows. In Section 2, we describe the BCOV propagator which is the building block of the graph integral. In Section 3, we define the graph integral considered in this paper. In Section 4, we prove the modular property of graph integrals. In Section 5, we prove that the graph integral has polynomial dependence in $\frac{1}{\operatorname{Im} \tau}$. In the appendix, we provide all the technical details of the necessary estimates for the graph integrals.

\section{BCOV propagator on the elliptic curve}

Let $\mathcal{H}=\{\tau \in \mathbb{C} \mid \operatorname{Im} \tau>0\}$ be the complex upper half-plane. Let $E_{\tau}=\mathbb{C} / \Lambda_{\tau}$ be the elliptic curve associated with the lattice

$$
\Lambda_{\tau}=\mathbb{Z} \oplus \tau \mathbb{Z}, \quad \tau \in \mathcal{H}
$$

We will use $z$ for the standard linear coordinate on $\mathbb{C}$, such that $E_{\tau}$ is obtained via the equivalence $z \sim z+1 \sim z+\tau$. The notation $d^{2} z$ will always refer to the following measure on $\mathbb{C}$ or $E_{\tau}$

$$
d^{2} z=\frac{\mathrm{i}}{2} d z \wedge d \bar{z}
$$


Let

$$
\Delta=-4 \frac{\partial}{\partial z} \frac{\partial}{\partial \bar{z}}
$$

be the standard flat Laplacian operator on $E_{\tau}$. We consider the kernel function $K_{t}^{E_{\tau}}$ for the heat operator $\mathrm{e}^{-t \Delta}$

$$
K_{t}^{E_{\tau}}\left(z_{1}, \bar{z}_{1} ;, z_{2}, \bar{z}_{2}\right)=\frac{1}{4 \pi t} \sum_{\lambda \in \Lambda_{\tau}} \mathrm{e}^{-\left|z_{1}-z_{2}+\lambda\right|^{2} / 4 t}, \quad t>0
$$

which is the unique function solving the heat equation

$$
\left(\frac{\partial}{\partial t}+\Delta_{z_{1}}\right) K_{t}^{E_{\tau}}\left(z_{1}, \bar{z}_{1} ;, z_{2}, \bar{z}_{2}\right)=0
$$

and the initial condition

$$
\lim _{t \rightarrow 0} \int_{E_{\tau}} d^{2} z_{2} K_{t}^{E_{\tau}}\left(z_{1}, \bar{z}_{1} ; z_{2}, \bar{z}_{2}\right) \phi\left(z_{2}, \bar{z}_{2}\right)=\phi\left(z_{1}, \bar{z}_{1}\right), \quad \forall \phi \in C^{\infty}\left(E_{\tau}\right) .
$$

Definition 2.1. The BCOV propagator $P_{\epsilon, L}^{E_{\tau}}$ is defined to be the smooth kernel function

$$
P_{\epsilon, L}^{E_{\tau}}\left(z_{1}, \bar{z}_{1} ; z_{2}, \bar{z}_{2}\right)=\int_{\epsilon}^{L} d t\left(\frac{\partial}{\partial z_{1}}\right)^{2} K_{t}^{E_{\tau}}\left(z_{1}, \bar{z}_{1} ;, z_{2}, \bar{z}_{2}\right), \quad \epsilon, L>0
$$

representing the operator $\int_{\epsilon}^{L} d t\left(\frac{\partial}{\partial z}\right)^{2} \mathrm{e}^{-t \Delta_{z}}$. We will also use $P_{0, \infty}^{E_{\tau}}\left(z_{1}, z_{2}\right)$ to represent the limit

$$
P_{0, \infty}^{E_{\tau}}\left(z_{1}, z_{2}\right) \equiv \lim _{\substack{\epsilon \rightarrow 0 \\ L \rightarrow \infty}} P_{\epsilon, L}^{E_{\tau}}\left(z_{1}, \bar{z}_{1} ;, z_{2}, \bar{z}_{2}\right)
$$

which is singular at $z_{1}=z_{2}$.

Note that we have dropped the anti-holomorphic dependence in $P_{0, \infty}^{E_{\tau}}$. It is shown in the next lemma that it is holomorphic away from the diagonal $z_{1}=z_{2}$. The kernel $P_{\epsilon, L}^{E_{\tau}}$ is motivated from string theory. It describes the propagator of the Kodaira-Spencer gauge theory, which is originally introduced in [2] on Calabi-Yau three-fold, and generalized in [3, 8] to Calabi-Yau manifolds of arbitrary dimensions.

The following Lemmas 2.1 and 2.2 for Green functions on elliptic curves are well known: 


\section{Lemma 2.1.}

$$
P_{0, \infty}^{E_{\tau}}\left(z_{1}, z_{2}\right)=\frac{1}{4 \pi} \wp\left(z_{1}-z_{2} ; \tau\right)+\frac{\pi}{12} E_{2}^{*}(\tau, \bar{\tau}), \quad \forall z_{1} \neq z_{2} .
$$

Here $\wp$ is the Weierstrass's elliptic function

$$
\begin{aligned}
\wp(z ; \tau) & =\frac{1}{z^{2}}+\sum_{\lambda \in \Lambda_{\tau}-\{0\}}\left(\frac{1}{(z-\lambda)^{2}}-\frac{1}{\lambda^{2}}\right) \\
E_{2}^{*}(\tau, \bar{\tau})=E_{2}(\tau)-\frac{3}{\pi \operatorname{Im} \tau}, \text { and } & \\
E_{2}(\tau) & =1-24 \sum_{n=1}^{\infty} \frac{n q^{n}}{1-q^{n}}, \quad q=\mathrm{e}^{2 \pi \mathrm{i} \tau}
\end{aligned}
$$

is the second Eisenstein series.

$P_{0, \infty}^{E_{\tau}}\left(z_{1}, z_{2}\right)$ becomes singular as $z_{1}$ approaches $z_{2}$, due to the singularity from the Green kernel. However, if we change the order of the limit, we have

\section{Lemma 2.2.}

$$
\lim _{\substack{\epsilon \rightarrow 0 \\ L \rightarrow \infty}} \lim _{z_{1} \rightarrow z_{2}} \partial_{z_{1}}^{n} P_{\epsilon, L}^{E_{\tau}}\left(z_{1}, z_{2}\right)= \begin{cases}\frac{\pi}{12} E_{2}^{*}, & \text { if } n=0 \\ \frac{(n+1) ! \zeta(n+2)}{2 \pi} E_{n+2}, & \text { if } n>0 \text { is even } \\ 0, & \text { if } n \text { is odd }\end{cases}
$$

where $E_{2 k}$ is the Eisenstein series of weight $2 k[9]$.

An elementary proof of Lemmas 2.1 and 2.2 is given in Appendix A.

The objects $\lim _{\substack{\epsilon \rightarrow 0 \\ L \rightarrow \infty}} \lim _{z_{1} \rightarrow z_{2}} \partial_{z_{1}}^{n} P_{\epsilon, L}^{E_{\tau}}\left(z_{1}, z_{2}\right)$ are special examples of the Feynman graph integrals to be discussed in the next section. They correspond to self-loops, and have nice modular properties. In fact, they are examples of almost holomorphic modular forms [7]. E $E_{2}^{*}$ plays a special role, which is modular but not holomorphic in $\tau$. However, its anti-holomorphic dependence is very mild, i.e., polynomial in $\frac{1}{\operatorname{Im} \tau}$. We will see that a large class of graph integrals will also have this property. 


\section{Feynman graph integral}

We consider a directed graph $\Gamma$. Let $V(\Gamma)$ be the set of vertices, $E(\Gamma)$ be the set of edges, and

$$
t, h: E \rightarrow V
$$

be the assignments of tail and head to each directed edge. We will also consider the decorated graph

$$
(\Gamma, n) \equiv\left(\Gamma,\left\{n_{e}\right\}_{e \in E}\right)
$$

where the decoration is given by

$$
n: E(\Gamma) \rightarrow \mathbb{Z}^{\geq 0}, \quad e \rightarrow n_{e},
$$

which associates each edge a non-negative integer. In the case that $n$ is the zero map, we will simply ignore $n$ and write $\Gamma$ for $(\Gamma, n)$.

Given a decorated graph $(\Gamma, n)$ and elliptic curve $E_{\tau}$, we associate the following graph integral:

$$
W_{(\Gamma, n)}\left(P_{\epsilon, L}^{E_{\tau}}\right)=\prod_{v \in V(\Gamma)} \int_{E_{\tau}} \frac{d^{2} z_{v}}{\operatorname{Im} \tau} \prod_{e \in E(\Gamma)}\left(\frac{\partial}{\partial z_{h(e)}}\right)^{n_{e}} P_{\epsilon, L ; e}^{E_{\tau}}
$$

where $P_{\epsilon, L ; e}^{E_{\tau}}=P_{\epsilon, L}^{E_{\tau}}\left(z_{h(e)}, \bar{z}_{h(e)} ; z_{t(e)}, \bar{z}_{t(e)}\right)$. The propagator $P_{\epsilon, L}^{E_{\tau}}$ is smooth as long as $\epsilon, L>0$, but exhibits singularity at the diagonal as $\epsilon \rightarrow 0$. However, the graph integral $W_{(\Gamma, n)}\left(P_{\epsilon, L}^{E_{\tau}}\right)$ has better behavior.

Lemma 3.1. The following limit exists

$$
\lim _{\substack{\epsilon \rightarrow 0 \\ L \rightarrow \infty}} W_{(\Gamma, n)}\left(P_{\epsilon, L}^{E_{\tau}}\right) .
$$

Proof. By Lemma 2.2, we can assume that $\Gamma$ is connected and has no selfloops. The singularity of $W_{(\Gamma, n)}\left(P_{\epsilon, L}^{E_{\tau}}\right)$ comes from the diagonals of the propagator as $\epsilon \rightarrow 0$. Let us fix $L$ first and analyze the limit $\epsilon \rightarrow 0$.

Let us first fix some notations. In the graph integral, we have associated a copy of $E_{\tau}$ for each $v \in V(\Gamma)$, which we will distinguish by $E_{v}$. Let $d$ be the distance function on $E_{\tau}$ with respect to the flat metric. Let $\chi:[0, \infty) \rightarrow[0,1]$ be a smooth function with $\chi(x)=1$ if $x<\delta$ and $\chi(x)=0$ if $x>2 \delta$, where 
$\delta \ll 0$ is a sufficient small positive number. Define

$$
K_{t}^{\delta}\left(z_{1}, \bar{z}_{1} ; z_{2}, \bar{z}_{2}\right)=\chi\left(d\left(z_{1}, z_{2}\right)^{2}\right) \frac{1}{4 t} \mathrm{e}^{-d\left(z_{1}, z_{2}\right)^{2} / 4 t}, \quad \forall z_{1}, z_{2} \in E_{\tau}
$$

and

$$
\hat{K}_{t}^{E_{\tau}}=K_{t}^{E_{\tau}}-K_{t}^{\delta}
$$

Then $\hat{K}_{t}^{E_{\tau}}$ is smooth as $t \rightarrow 0$. Similarly, we define

$$
P_{\epsilon, L}^{\delta}\left(z_{1}, \bar{z}_{1} ; z_{2}, \bar{z}_{2}\right)=\int_{\epsilon}^{L} d t \partial_{z_{1}}^{2} K_{t}^{\delta}\left(z_{1}, \bar{z}_{1} ; z_{2}, \bar{z}_{2}\right), \quad \hat{P}_{\epsilon, L}^{\delta}=P_{\epsilon, L}^{E_{\tau}}-P_{\epsilon, L}^{\delta} .
$$

$\hat{P}_{\epsilon, L}^{\delta}$ is smooth as $\epsilon \rightarrow 0$ and $P_{\epsilon, L}^{\delta}$ contains all the information about the singularity.

The graph integral $W_{(\Gamma, n)}\left(P_{\epsilon, L}^{E_{\tau}}\right)=W_{(\Gamma, n)}\left(P_{\epsilon, L}^{\delta}+\hat{P}_{\epsilon, L}^{\delta}\right)$ splits into a sum of graph integrals where we associate $P_{\epsilon, L}^{\delta}$ or $\hat{P}_{\epsilon, L}^{\delta}$ on each edge. Let us pick up a particular term, and let $\Gamma^{\prime}$ be the corresponding subgraph of $\Gamma$ consisting of those edges assigned with the singular propagator $P_{\epsilon, L}^{\delta}$. Let $\Gamma^{\prime}=\Gamma_{1} \cup \cdots \cup \Gamma_{k}$ be the decomposition into connected components. It is sufficient to show that each connected component $\Gamma_{i}$ contributes a regular integral as $\epsilon \rightarrow 0$.

Let us focus on one component $\Gamma_{1}$. Let $v_{\bullet} \in V\left(\Gamma_{1}\right)$ be an arbitrary vertex. The integral is supported near the diagonal of $\prod_{v \in V\left(\Gamma_{1}\right)} E_{v}$, which can be identified with a small neighborhood of zero section of the vector bundle $T_{E_{v}}^{\oplus\left(\left|V\left(\Gamma_{1}\right)\right|-1\right)} \cong E_{v_{\bullet}} \times \mathbb{C}^{\oplus\left(\left|V\left(\Gamma_{1}\right)\right|-1\right)}$ on $E_{v_{\bullet}}$. Here $T_{E_{v}}$ is the tangent bundle of $E_{v_{\bullet}}$. Therefore we can write the relevant graph integral on $\Gamma_{1}$ into the form

$$
\int_{E_{v_{\bullet}}} d^{2} z_{v_{\bullet}} \prod_{v \in V\left(\Gamma_{1}\right) \backslash\left\{v_{\bullet}\right\}} \int_{\mathbb{C}} d^{2} y_{v}\left(\prod_{e \in E\left(\Gamma_{1}\right)} \partial_{y_{e}}^{m_{e}} H_{\epsilon}^{L}\left(y_{e}, \bar{y}_{e}\right)\right) \Phi,
$$

where $H_{\epsilon}^{L}(z, \bar{z})=\int_{\epsilon}^{L} \frac{d t}{4 \pi t} \mathrm{e}^{-|z|^{2}} / 4 t, m_{e}$ some non-negative integers for each edge $e \in E\left(\Gamma_{1}\right)$,

$$
y_{e}= \begin{cases}y_{h(e)}, & \text { if } t(e)=v_{\bullet} \\ -y_{t(e)}, & \text { if } h(e)=v_{\bullet} \\ y_{h(e)}-y_{t(e)}, & \text { otherwise }\end{cases}
$$


and $\Phi$ is a smooth function on $E_{v_{\bullet}} \times \mathbb{C}^{\oplus\left(\left|V\left(\Gamma_{1}\right)\right|-1\right)} \times \prod_{v \notin V\left(\Gamma_{1}\right)} E_{v}$ with compact support. By Proposition B.1 and its proof, the above integral is regular and uniformly convergent as $\epsilon \rightarrow 0$. This proves that $\lim _{\epsilon \rightarrow 0} W_{(\Gamma, n)}\left(P_{\epsilon, L}^{E_{\tau}}\right)$ exists.

Now we consider the limit $L \rightarrow \infty$. Since $P_{\epsilon, \infty}^{E_{\tau}}=P_{\epsilon, L}^{E_{\tau}}+P_{L, \infty}^{E_{\tau}}$ and the kernel function $P_{L, \infty}^{E_{\tau}}$ is smooth. It follows that

$$
\lim _{\epsilon \rightarrow 0} W_{(\Gamma, n)}\left(P_{\epsilon, \infty}^{E_{\tau}}\right)=\lim _{\epsilon \rightarrow 0} W_{(\Gamma, n)}\left(P_{\epsilon, L}^{E_{\tau}}+P_{L, \infty}^{E_{\tau}}\right)
$$

exits. This proves the lemma.

Definition 3.1. Given a decorated graph $(\Gamma, n)$, we define a smooth function $W_{(\Gamma, n)}$ on $\mathcal{H}$ by

$$
W_{(\Gamma, n)}(\tau, \bar{\tau}) \equiv \lim _{\substack{\epsilon \rightarrow 0 \\ L \rightarrow \infty}} W_{(\Gamma, n)}\left(P_{\epsilon, L}^{E_{\tau}}\right)
$$

Example 3.1. Consider the self-loop graph with decoration $n$.

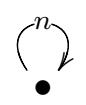

Lemma 2.2 implies that

$$
W_{n}= \begin{cases}\frac{\pi}{12} E_{2}^{*}, & \text { if } n=0, \\ \frac{(n+1) ! \zeta(n+2)}{2 \pi} E_{n+2}, & \text { if } n>0 \text { is even } \\ 0, & \text { if } n \text { is odd. }\end{cases}
$$

\section{Modularity}

We consider the modular group $S L(2 . \mathbb{Z})$, which acts on $\mathcal{H}$ by

$$
\tau \rightarrow \gamma \tau=\frac{A \tau+B}{C \tau+D}, \quad \text { for } \gamma \in\left(\begin{array}{ll}
A & B \\
C & D
\end{array}\right) \in S L(2, \mathbb{Z}) .
$$

Recall that a function $f: \mathcal{H} \rightarrow \mathbb{C}$ is said to have weight $k$ under the modular group $S L(2, \mathbb{Z})$ if

$$
f(\gamma \tau)=(C \tau+D)^{k} f(\tau) \text { for all } \gamma \in\left(\begin{array}{ll}
A & B \\
C & D
\end{array}\right) \in S L(2, \mathbb{Z}) .
$$


Proposition 4.1. The graph integral $W_{(\Gamma, n)}(\tau, \bar{\tau})$ has weight $\sum_{e \in E(\Gamma)}\left(n_{e}+\right.$ 2) under $S L(2, \mathbb{Z})$.

Proof. Given $\gamma \in\left(\begin{array}{ll}A & B \\ C & D\end{array}\right) \in S L(2, \mathbb{Z})$, the lattice transforms as $\Lambda_{\gamma \tau}=$ $\frac{1}{(C \tau+D)} \Lambda_{\tau}$. It follows that the propagator has the transformation property

$$
\begin{aligned}
& \partial_{z_{1}}^{m} P_{\epsilon, L}^{E_{\gamma \tau}}\left(z_{1}, \bar{z}_{1} ;, z_{2}, \bar{z}_{2}\right) \\
& \quad=(C \tau+D)^{m+2}\left(\partial_{z_{1}}^{m} P_{|C \tau+D|^{2} \epsilon,|C \tau+D|^{2} L}^{E_{\tau}}\right)\left(z_{1}^{\prime}, \bar{z}_{1}^{\prime} ; z_{2}^{\prime}, \bar{z}_{2}^{\prime}\right)
\end{aligned}
$$

where $z_{i}^{\prime}=(C \tau+D) z_{i}, i=1,2$. Using the modular invariance of the measure $\frac{d^{2} z}{\operatorname{Im} \tau}$, we find

$$
\begin{aligned}
W_{(\Gamma, n)}\left(P_{\epsilon, L}^{E_{\gamma \tau}}\right)= & \prod_{v \in V(\Gamma)} \int_{E_{\gamma \tau}} \frac{d^{2} z_{v}}{\operatorname{Im}(\gamma \tau)} \\
& \times \prod_{e \in E(\Gamma)}\left(\partial_{z_{h(e)}}^{n_{e}} P_{\epsilon, L}^{E_{\gamma \tau}}\right)\left(z_{h(e)}, \bar{z}_{h(e)} ; z_{t(e)}, \bar{z}_{t(e)}\right) \\
= & \prod_{v \in V(\Gamma)} \int_{E_{\tau}} \frac{d^{2} z_{v}}{\operatorname{Im} \tau} \prod_{e \in E(\Gamma)}(C \tau+D)^{n_{e}+2} \partial_{z_{h(e)}}^{n_{e}} \\
& \times P_{|C \tau+D|^{2} \epsilon,|C \tau+D|^{2} L}^{E_{\tau}}\left(z_{h(e)}, \bar{z}_{h(e)} ; z_{t(e)}, \bar{z}_{t(e)}\right) \\
= & (C \tau+D)^{\sum_{e \in E(\Gamma)}\left(n_{e}+2\right)} W_{(\Gamma, n)}\left(P_{|C \tau+D|^{2} \epsilon,|C \tau+D|^{2} L}^{E_{\tau}}\right) .
\end{aligned}
$$

The proposition follows after taking the limit $\epsilon \rightarrow 0, L \rightarrow \infty$.

\section{Anti-holomorphic dependence}

The function $W_{(\Gamma, n)}$ has particular weight under modular transformation. However, it's not holomorphic in general. We have seen this when $\Gamma$ is a one-vertex graph with a self-loop. In this case $W_{\Gamma}=\frac{\pi}{12} E_{2}^{*}$, which exhibits a polynomial dependence on $\frac{1}{\operatorname{Im} \tau}$. In this section, we will show that the $\bar{\tau}$ dependence of any graph integral is polynomial in $\frac{1}{\operatorname{Im} \tau}$.

Proposition 5.1. For any decorated graph $(\Gamma, n)$, the graph integral can be decomposed as

$$
W_{(\Gamma, n)}(\tau, \bar{\tau})=\sum_{i=0}^{N} f_{i}(\tau) \frac{1}{(\operatorname{Im} \tau)^{i}}
$$


where $f_{i}(\tau)$ 's are holomorphic functions on $\mathcal{H}$, and $N$ is some non-negative integer.

Proof. We will show that $\partial_{\bar{\tau}} W_{(\Gamma, n)}(\tau, \bar{\tau})$ is also some graph integral with fewer edges. The proposition will then follow by induction. Without loss of generality, we can assume that $\Gamma$ has no self-loops.

First of all, it's easy to see that

$$
\frac{\partial}{\partial \bar{\tau}} \int_{E_{\tau}} \frac{d^{2} z_{\tau}}{\operatorname{Im} \tau} f(z, \bar{z} ; \tau, \bar{\tau})=\int_{E_{\tau}} \frac{d^{2} z_{\tau}}{\operatorname{Im} \tau}\left(\frac{\operatorname{Im} z}{\operatorname{Im} \tau} \frac{\partial}{\partial \bar{z}}+\frac{\partial}{\partial \bar{\tau}}\right) f(z, \bar{z} ; \tau, \bar{\tau}) .
$$

Here the integration on $E_{\tau}$ is performed in the region $\{a+b \tau \mid 0 \leq a, b \leq 1\}$. Hence

$$
\begin{aligned}
\partial_{\bar{\tau}} W_{(\Gamma, n)}\left(P_{\epsilon, L}^{E_{\tau}}\right)= & \prod_{v \in V(\Gamma)} \int_{E_{\tau}} \frac{d^{2} z_{v}}{\operatorname{Im} \tau} \\
& \times \sum_{e \in E(\Gamma)}\left[\left(\frac{\operatorname{Im}\left(z_{h(e)}-z_{t(e)}\right)}{\operatorname{Im} \tau} \frac{\partial}{\partial \bar{z}_{h(e)}}+\frac{\partial}{\partial \bar{\tau}}\right) \partial_{z_{h(e)}}^{n_{e}} P_{\epsilon, L, e}^{E_{\tau}}\right] \\
& \times\left(\prod_{e^{\prime} \in E(\Gamma) \backslash\{e\}} \partial_{z_{h\left(e^{\prime}\right)}^{n_{e^{\prime}}}}^{E_{\epsilon, L, e^{\prime}}}\right)
\end{aligned}
$$

To simplify the notation, we will write

$$
z_{e} \equiv z_{h(e)}-z_{t(e)}
$$

for any $e \in E(\Gamma)$. Using the heat equation, we find

$$
\begin{aligned}
& \left(\frac{\operatorname{Im}\left(z_{h(e)}-z_{t(e)}\right)}{\operatorname{Im} \tau} \frac{\partial}{\partial \bar{z}_{h(e)}}+\frac{\partial}{\partial \bar{\tau}}\right) \partial_{z_{h(e)}}^{n_{e}} P_{\epsilon, L, e}^{E_{\tau}} \\
& \quad=\left.\sum_{\lambda \in \Lambda_{\tau}} \frac{\operatorname{Im}\left(z_{e}-\lambda\right)}{\operatorname{Im} \tau} \frac{1}{16 \pi t} \partial_{z_{e}}^{n_{e}+1} \mathrm{e}^{-\left|z_{e}-\lambda\right|^{2} / 4 t}\right|_{t=\epsilon} ^{t=L}
\end{aligned}
$$

$\partial_{\bar{\tau}} W_{(\Gamma, n)}\left(P_{\epsilon, L}^{E_{\tau}}\right)$ has two types of contributions corresponding to $t=\epsilon$ or $t=L$ in the above formula. 
The term with $\boldsymbol{t}=\boldsymbol{L} \quad$ Let us first consider the term with $t=L$. If $n_{e}>0$, then the summation $\sum_{\lambda \in \Lambda_{\tau}} \frac{\operatorname{Im}\left(z_{e}-\lambda\right)}{\operatorname{Im} \tau} \frac{1}{16 \pi t} \partial_{z_{e}}^{n_{e}+1} \mathrm{e}^{-\left|z_{e}-\lambda\right|^{2} / 4 t}$ is absolutely convergent and uniform in $t$, so

$$
\lim _{L \rightarrow \infty} \sum_{\lambda \in \Lambda_{\tau}} \frac{\operatorname{Im}\left(z_{e}-\lambda\right)}{\operatorname{Im} \tau} \frac{1}{16 \pi L} \partial_{z_{e}}^{n_{e}+1} \mathrm{e}^{-\left|z_{e}-\lambda\right|^{2} / 4 L}=0
$$

If $n_{e}=0$, then

$$
\begin{aligned}
\sum_{\lambda \in \Lambda_{\tau}} & \frac{\operatorname{Im}\left(z_{e}-\lambda\right)}{\operatorname{Im} \tau} \frac{1}{16 \pi L} \partial_{z_{e}} \mathrm{e}^{-\left|z_{e}-\lambda\right|^{2} / 4 L} \\
= & \sum_{n \in \mathbb{Z}}\left(\frac{\operatorname{Im} z_{e}}{\operatorname{Im} \tau}-n\right) \sum_{m \in \mathbb{Z}}\left(\frac{\bar{z}_{e}-(m+n \bar{\tau})}{64 \pi L^{2}}\right) \mathrm{e}^{-\left|z_{e}-(m+n \tau)\right|^{2} / 4 L} \\
= & \sum_{n \in \mathbb{Z}}\left(\frac{\operatorname{Im} z_{e}}{\operatorname{Im} \tau}-n\right) \sum_{m \in \mathbb{Z}}\left[\left(\frac{\bar{z}_{e}-(m+n \bar{\tau})}{64 \pi L^{2}}\right) \mathrm{e}^{-\left|z_{e}-(m+n \tau)\right|^{2} / 4 L}\right. \\
& \left.-\int_{m}^{m+1} d y\left(\frac{\bar{z}_{e}-(y+n \bar{\tau})}{64 \pi L^{2}}\right) \mathrm{e}^{-\left|z_{e}-(y+n \tau)\right|^{2} / 4 L}\right] \\
& +\sum_{n \in \mathbb{Z}}\left(\frac{\operatorname{Im} z_{e}}{\operatorname{Im} \tau}-n\right) \int_{-\infty}^{\infty} d y\left(\frac{\bar{z}_{e}-(y+n \bar{\tau})}{64 \pi L^{2}}\right) \mathrm{e}^{-\left|z_{e}-(y+n \tau)\right|^{2} / 4 L} \\
= & I_{1}+I_{2} .
\end{aligned}
$$

Similarly we have $\lim _{L \rightarrow \infty} I_{1}=0 . I_{2}$ can be computed using Gaussian integral

$$
\begin{aligned}
I_{2} & =\sum_{n \in \mathbb{Z}}\left(\frac{\operatorname{Im} z_{e}}{\operatorname{Im} \tau}-n\right)\left(\frac{\operatorname{Im} z_{e}-n \operatorname{Im} \tau}{32 i \sqrt{\pi} L^{3 / 2}}\right) \mathrm{e}^{-\left(\operatorname{Im} z_{e}-n \operatorname{Im} \tau\right)^{2} / 4 L} \\
& =\frac{1}{32 \mathrm{i} \sqrt{\pi}(\operatorname{Im} \tau)^{2}} \sum_{n \in \mathbb{Z}} \frac{\left(\frac{\operatorname{Im} z_{e}}{\operatorname{Im} \tau}-n\right)^{2}}{\tilde{L}^{3 / 2}} \mathrm{e}^{-\left(\frac{\operatorname{Im} z_{e}}{\operatorname{Im} \tau}-n\right)^{2} / 4 \tilde{L}}, \quad \text { where } \tilde{L}=\frac{L}{(\operatorname{Im} \tau)^{2}} \\
& =\frac{1}{8 \mathrm{i}(\operatorname{Im} \tau)^{2}} \sum_{m \in \mathbb{Z}}\left(1-8 \tilde{L} \pi^{2} m^{2}\right) \mathrm{e}^{-4 m^{2} \pi^{2} \tilde{L}+2 \pi i m \frac{\operatorname{Im} z_{e}}{\operatorname{Im} \tau}},
\end{aligned}
$$

where in the last step we have used Fourier transformation. Therefore

$$
\lim _{L \rightarrow \infty} I_{2}=\frac{1}{8 \mathrm{i}(\operatorname{Im} \tau)^{2}}
$$


To summarize, we find

$$
\lim _{L \rightarrow \infty} \sum_{\lambda \in \Lambda_{\tau}} \frac{\operatorname{Im}\left(z_{e}-\lambda\right)}{\operatorname{Im} \tau} \frac{1}{16 \pi L} \partial_{z_{e}}^{n_{e}+1} \mathrm{e}^{-\left|z_{e}-\lambda\right|^{2} / 4 L}= \begin{cases}\frac{1}{8 i(\operatorname{Im} \tau)^{2}}, & \text { if } n_{e}=0 \\ 0, & \text { if } n_{e}>0\end{cases}
$$

The term with $\boldsymbol{t}=\boldsymbol{\epsilon} \quad$ Now we consider the term with $t=\epsilon$. Its contribution to $\partial_{\bar{\tau}} W_{(\Gamma, n)}$ is

$$
\begin{aligned}
\prod_{v \in V(\Gamma)} & \int_{E_{\tau}} \frac{d^{2} z_{v}}{\operatorname{Im} \tau} \sum_{e \in E(\Gamma)}\left(\sum_{\lambda \in \Lambda_{\tau}} \frac{\operatorname{Im}\left(z_{e}-\lambda\right)}{\operatorname{Im} \tau} \frac{1}{16 \pi \epsilon} \partial_{z_{e}}^{n_{e}+1} e^{-\left|z_{e}-\lambda\right|^{2} / 4 \epsilon}\right) \\
& \times\left(\prod_{e^{\prime} \in E(\Gamma) \backslash\{e\}} \partial_{z_{h\left(e^{\prime}\right)}^{n_{e^{\prime}}} P_{\epsilon, L ; e^{\prime}}^{E_{\tau}}}^{E^{\prime}}\right) \\
= & \sum_{e \in E(\Gamma)}\left(\prod_{v \in V(\Gamma) \backslash\{h(e)\}} \int_{E_{\tau}} \frac{d^{2} z_{v}}{\operatorname{Im} \tau}\right) \int_{\mathbb{C} \frac{d^{2} z_{h(e)}}{(\operatorname{Im} \tau)^{2}}} \\
& \times\left(\frac{\operatorname{Im} z_{e}}{16 \pi \epsilon} \partial_{z_{e}}^{n_{e}+1} e^{-\left|z_{e}\right|^{2} / 4 \epsilon}\right)\left(\prod_{e^{\prime} \in E(\Gamma) \backslash\{e\}} \partial_{z_{h\left(e^{\prime}\right)}^{n_{e^{\prime}}} P_{\epsilon, L ; e^{\prime}}^{E_{\tau}}}\right)
\end{aligned}
$$

By Proposition B.2, it reduces to certain graph integral on $\Gamma^{\prime}$ under the limit $\epsilon \rightarrow 0, L \rightarrow \infty$, with an extra factor proportional to $\frac{1}{(\operatorname{Im} \tau)^{2}}$. Here $\Gamma^{\prime}$ is obtained from $\Gamma$ by collapsing one edge.

Combining the terms for $t=L$ and terms for $t=\epsilon$, it follows by induction that

$$
\partial_{\bar{\tau}} W_{(\Gamma, n)}=\lim _{\substack{\epsilon \rightarrow 0 \\ L \rightarrow \infty}} \partial_{\bar{\tau}} W_{(\Gamma, n)}\left(P_{\epsilon, L}^{E_{\tau}}\right)=\frac{1}{(\operatorname{Im} \tau)^{2}} \sum_{i=0}^{K} f_{i}(\tau) \frac{1}{(\operatorname{Im} \tau)^{i}}
$$

for some holomorphic function $f_{i}(\tau)$ and non-negative integer $K$. Therefore $W_{(\Gamma, n)}$ has polynomial dependence on $\frac{1}{\operatorname{Im} \tau}$ as well.

Corollary 5.1. Let $\Gamma$ be a graph such that every two vertices are connected by at most one edge, then

$$
\partial_{\bar{\tau}} W_{\Gamma}=\frac{\mathrm{i}}{8(\operatorname{Im} \tau)^{2}} \sum_{e \in E(\Gamma)}\left(W_{\Gamma / e}-W_{\Gamma \backslash e}\right)
$$

where $\Gamma / e$ is the graph by collapsing the edge $e$ in $\Gamma$, and $\Gamma \backslash e$ is the graph by deleting the edge e in $\Gamma$. 
Proof. In the proof of Proposition 5.1, there are two contributions. The term with $L \rightarrow \infty$ contributes $\frac{1}{8 \mathrm{i}(\operatorname{Im} \tau)^{2}} W_{\Gamma \backslash e}$. The term with $\epsilon \rightarrow 0$ comes from the integration

$$
\int_{\mathbb{C}} \frac{d^{2} z_{h(e)}}{(\operatorname{Im} \tau)^{2}}\left(\frac{\operatorname{Im} z_{e}}{16 \pi \epsilon} \partial_{z_{e}} \mathrm{e}^{-\left|z_{e}\right|^{2} / 4 \epsilon}\right)=\frac{\mathrm{i}}{8(\operatorname{Im} \tau)^{2}} \int_{\mathbb{C}} d^{2} z_{h(e)} \frac{1}{4 \pi \epsilon} \mathrm{e}^{-\left|z_{e}\right|^{2} / 4 \epsilon},
$$

which becomes $\delta$-distribution $\frac{\mathrm{i}}{8(\operatorname{Im} \tau)^{2}} \delta_{z_{e}, 0}$ as $\epsilon \rightarrow 0$.

Proposition 5.2. For any decorated graph $(\Gamma, n)$, the graph integral $W_{(\Gamma, n)}(\tau, \bar{\tau})$ is an almost modular form of weight $\sum_{e \in E(\Gamma)}\left(n_{e}+2\right)$.

Proof. This follows from Propositions 4.1 and 5.1.

Theorem 1.1 from the introduction follows as a special case for undecorated graphs (i.e., $n_{e}=0$ for all edges).

\section{Acknowledgment}

The author thanks K. Costello for many stimulating discussions on two dimensional quantum field theory, and thanks S.T. Yau for useful conversations on quasi-modular forms.

\section{Appendix A. The BCOV propagator}

In this section, we give an elementary proof of Lemmas 2.1 and 2.2 .

Proof of Lemma 2.1. Let $z_{12}=z_{1}-z_{2}$.

$$
\begin{aligned}
P_{\epsilon, L}^{E_{\tau}}\left(z_{1}, \bar{z}_{1} ; z_{2}, \bar{z}_{2}\right)= & \int_{\epsilon}^{L} \frac{d t}{4 \pi t} \sum_{m, n \in \mathbb{Z}}\left(\frac{\bar{z}_{12}-(m+n \bar{\tau})}{4 t}\right)^{2} \\
& \times \exp \left(-\left|z_{12}-(m+n \tau)\right|^{2} / 4 t\right) \\
= & \int_{\epsilon}^{L} \frac{d t}{4 \pi t} \sum_{m \in \mathbb{Z}}\left(\frac{\bar{z}_{12}-m}{4 t}\right)^{2} \exp \left(-\left|z_{12}-m\right|^{2} / 4 t\right) \\
& +\int_{\epsilon}^{L} \frac{d t}{4 \pi t} \sum_{n \neq 0} \sum_{m \in \mathbb{Z}}\left[\left(\frac{\bar{z}_{12}-(m+n \bar{\tau})}{4 t}\right)^{2}\right.
\end{aligned}
$$




$$
\begin{aligned}
& \times \exp \left(-\left|z_{12}-(m+n \tau)\right|^{2} / 4 t\right) \\
& -\int_{m}^{m+1} d y\left(\frac{\bar{z}_{12}-(y+n \bar{\tau})}{4 t}\right)^{2} \\
& \left.\times \exp \left(-\left|z_{12}-(y+n \tau)\right|^{2} / 4 t\right)\right] \\
& +\int_{\epsilon}^{L} \frac{d t}{4 \pi t} \sum_{n \neq 0} \int_{-\infty}^{\infty} d y\left(\frac{\bar{z}_{12}-(y+n \bar{\tau})}{4 t}\right)^{2} \\
& \times \exp \left(-\left|z_{12}-(y+n \tau)\right|^{2} / 4 t\right) \\
& =I_{1}+I_{2}+I_{3} .
\end{aligned}
$$

$I_{1}$ is absolutely convergent and

$$
\begin{aligned}
\lim _{\substack{t \rightarrow 0 \\
L \rightarrow \infty}} I_{1} & =\int_{0}^{\infty} \frac{d t}{4 \pi t} \sum_{m \in \mathbb{Z}}\left(\frac{\bar{z}_{12}-m}{4 t}\right)^{2} \exp \left(-\left|z_{12}-m\right|^{2} / 4 t\right) \\
& =\sum_{m \in \mathbb{Z}} \frac{1}{\left(z_{12}-m\right)^{2}} \int_{0}^{\infty} \frac{d t}{4 \pi t} \frac{1}{(4 t)^{2}} \exp (-1 / 4 t) \\
& =\frac{1}{4 \pi} \sum_{m \in \mathbb{Z}} \frac{1}{\left(z_{12}-m\right)^{2}} .
\end{aligned}
$$

$I_{2}$ is also absolutely convergent. To see this, let

$$
\begin{aligned}
F(y) & =\left(\frac{\bar{z}_{12}-(y+n \bar{\tau})}{4 t}\right)^{2} \exp \left(-\left|z_{12}-(y+n \tau)\right|^{2} / 4 t\right) \\
& =\frac{1}{\left(z_{12}-y-n \tau\right)^{2}} G(u), \quad u=t /\left|z_{12}-(y+n \tau)\right|^{2}
\end{aligned}
$$

where $G(u)=\frac{1}{(4 u)^{2}} \exp (-1 / 4 u)$ which is a smooth and bounded function on $[0, \infty)$. Since

$$
\begin{aligned}
\frac{d F(y)}{d y}= & \frac{2}{\left(z_{12}-y-n \tau\right)^{3}} G(u) \\
& +\left(\frac{1}{\left(z_{12}-y-n \tau\right)^{3}}+\frac{1}{\left(z_{12}-y-n \tau\right)^{2}\left(\bar{z}_{12}-y-n \bar{\tau}\right)}\right) u G^{\prime}(u)
\end{aligned}
$$

We can write $I_{2}$ as

$$
I_{2}=\int_{\epsilon}^{L} \frac{d t}{4 \pi t} \sum_{n \neq 0} \sum_{m \in Z} \int_{m}^{m+1} d y(F(m)-F(y)),
$$


which is of the order $\frac{1}{|m+n \tau|^{3}}$ as $m, n \rightarrow \infty$. Therefore similar to the calculation for $I_{1}$, we find

$$
\begin{aligned}
\lim _{\substack{\epsilon \rightarrow 0 \\
L \rightarrow \infty}} I_{2} & =\frac{1}{4 \pi} \sum_{n \neq 0} \sum_{m \in \mathbb{Z}}\left(\frac{1}{\left(z_{12}-m-n \tau\right)^{2}}-\int_{m}^{m+1} d y \frac{1}{\left(z_{12}-y-n \tau\right)^{2}}\right) \\
& =\frac{1}{4 \pi} \sum_{n \neq 0} \sum_{m \in \mathbb{Z}} \frac{1}{\left(z_{12}-m-n \tau\right)^{2}} .
\end{aligned}
$$

To evaluate $I_{3}$, notice that

$$
\begin{aligned}
& \int_{-\infty}^{\infty} d y\left(\frac{\bar{z}_{12}-(y+n \bar{\tau})}{4 t}\right)^{2} \exp \left(-\left|z_{12}-(y+n \tau)\right|^{2} / 4 t\right) \\
& \quad=\int_{-\infty}^{\infty} d y \frac{y^{2}-\left(\operatorname{Im} z_{12}-n \operatorname{Im} \tau\right)^{2}}{(4 t)^{2}} \exp \left(-y^{2} / 4 t-\left(\operatorname{Im} z_{12}-n \operatorname{Im} \tau\right)^{2} / 4 t\right) \\
& \quad=-\frac{\sqrt{\pi}\left(\left(\operatorname{Im} z_{12}-n \operatorname{Im} \tau\right)^{2} / t-2\right)}{8 t^{1 / 2}} \exp \left(-\left(\operatorname{Im} z_{12}-n \operatorname{Im} \tau\right)^{2} / 4 t\right) \\
& =t \frac{d}{d t}\left(-\frac{\pi}{(4 \pi t)^{1 / 2}} \exp \left(-\left(\operatorname{Im} z_{12}-n \operatorname{Im} \tau\right)^{2} / 4 t\right)\right)
\end{aligned}
$$

Therefore,

$$
\begin{aligned}
\lim _{\substack{\epsilon \rightarrow 0 \\
L \rightarrow \infty}} I_{3} & =-\left.\lim _{\substack{\epsilon \rightarrow 0 \\
L \rightarrow \infty}} \frac{1}{4} \sum_{n \neq 0}\left(\frac{1}{(4 \pi t)^{1 / 2}} \exp \left(-\left(\operatorname{Im} z_{12}-n \operatorname{Im} \tau\right)^{2} / 4 t\right)\right)\right|_{\epsilon} ^{L} \\
& =-\left.\lim _{\substack{\epsilon \rightarrow 0 \\
L \rightarrow \infty}} \frac{1}{4 \operatorname{Im} \tau} \sum_{n \neq 0}\left(\frac{1}{(4 \pi t)^{1 / 2}} \exp \left(-(a-n)^{2} / 4 t\right)\right)\right|_{\epsilon} ^{L} \\
a & =\operatorname{Im} z_{12} / \operatorname{Im} \tau, 0 \leq a<1 .
\end{aligned}
$$

Obviously,

$$
\lim _{\epsilon \rightarrow 0} \sum_{n \neq 0}\left(\frac{1}{(4 \pi \epsilon)^{1 / 2}} \exp \left(-(a-n)^{2} / 4 \epsilon\right)\right)=0 .
$$

The Poisson summation formula gives

$$
\sum_{n \in \mathbb{Z}}\left(\frac{1}{(4 \pi L)^{1 / 2}} \exp \left(-(a-n)^{2} / 4 L\right)\right)=\sum_{m \in \mathbb{Z}} \exp \left(-4 \pi^{2} m^{2} L+2 \pi \mathrm{i} m a\right)
$$


hence

$$
\begin{aligned}
& \lim _{L \rightarrow \infty} \sum_{n \in \mathbb{Z}}\left(\frac{1}{(4 \pi L)^{1 / 2}} \exp \left(-(a-n)^{2} / 4 L\right)\right) \\
& \quad=\lim _{L \rightarrow \infty} \sum_{m \in \mathbb{Z}} \exp \left(-4 \pi^{2} m^{2} L+2 \pi \mathrm{i} m a\right)=1 .
\end{aligned}
$$

Adding the three terms together, we find

$$
\begin{aligned}
\lim _{\substack{\epsilon \rightarrow 0 \\
L \rightarrow \infty}}\left(P_{\epsilon, L}^{E_{\tau}}\left(z_{1}, \bar{z}_{1} ; z_{2}, \bar{z}_{2}\right)\right) & =\frac{1}{4 \pi} \sum_{n \in \mathbb{Z}} \sum_{m \in \mathbb{Z}} \frac{1}{\left(z_{12}-m-n \tau\right)^{2}}-\frac{1}{4 \operatorname{Im} \tau} \\
& =\frac{1}{4 \pi} \wp\left(z_{12} ; \tau\right)+\frac{\pi}{12} E_{2}(\tau)-\frac{1}{4 \operatorname{Im} \tau} \\
& =\frac{1}{4 \pi} \wp\left(z_{12} ; \tau\right)+\frac{\pi}{12} E_{2}^{*}(\tau ; \bar{\tau}) .
\end{aligned}
$$

Proof of Lemma 2.2. From the proof of Lemma 2.1, it's easy to see that

$$
\begin{aligned}
& \lim _{\substack{\epsilon \rightarrow 0 \\
L \rightarrow \infty}} \lim _{z_{1} \rightarrow z_{2}} I_{1}\left(z_{1}, z_{2}\right)=\frac{1}{4 \pi} \sum_{m \neq 0} \frac{1}{m^{2}}, \\
& \lim _{\substack{\epsilon \rightarrow 0 \\
L \rightarrow \infty}} \lim _{z_{1} \rightarrow z_{2}} I_{2}\left(z_{1}, z_{2}\right)=\frac{1}{4 \pi} \sum_{n \neq 0} \sum_{m \in Z} \frac{1}{(m+n \tau)^{2}}, \\
& \lim _{\substack{\epsilon \rightarrow 0 \\
L \rightarrow \infty}} \lim _{z_{1} \rightarrow z_{2}} I_{3}\left(z_{1}, z_{2}\right)=-\frac{1}{4 \operatorname{Im} \tau} .
\end{aligned}
$$

It follows that

$$
\lim _{\substack{\epsilon \rightarrow 0 \\ L \rightarrow \infty}} \lim _{z_{1} \rightarrow z_{2}} P_{\epsilon, L}^{E_{\tau}}\left(z_{1}, z_{2}\right)=\frac{\pi}{12} E_{2}^{*}(\tau, \bar{\tau}) .
$$

This proves the case for $n=0$. For $n>0$,

$$
\begin{aligned}
\partial_{z_{1}}^{n} & P_{\epsilon, L}^{E_{\tau}}\left(z_{1}, z_{2}\right) \\
= & \int_{\epsilon}^{L} \frac{d t}{4 \pi t} \sum_{m, n \in \mathbb{Z}}\left(\frac{\bar{z}_{12}-(m+n \bar{\tau})}{4 t}\right)^{n+2} \exp \left(-\left|z_{12}-(m+n \tau)\right|^{2} / 4 t\right) \\
= & \int_{\epsilon}^{L} \frac{d t}{4 \pi t} \sum_{m, n \in \mathbb{Z}} \frac{1}{\left(z_{12}-(m+n \tau)\right)^{n+2}} \frac{\left|\bar{z}_{12}-(m+n \bar{\tau})\right|^{2}}{4 t} \\
& \times \exp \left(-\left|z_{12}-(m+n \tau)\right|^{2} / 4 t\right),
\end{aligned}
$$


which is in fact absolutely convergent. Therefore in this case

$$
\begin{aligned}
\lim _{\substack{\epsilon \rightarrow 0 \\
L \rightarrow \infty}} \lim _{z_{1} \rightarrow z_{2}} \partial_{z_{1}}^{n} P_{\epsilon, L}^{E_{\tau}}\left(z_{1}, z_{2}\right) & =\sum_{\substack{m, n \in \mathbb{Z} \\
(m, n) \neq(0,0)}} \frac{1}{(m+n \tau)^{n+2}} \int_{0}^{\infty} \frac{d t}{4 \pi t} \frac{1}{t^{n+2}} \mathrm{e}^{-1 / t} \\
& =\frac{(n+1) !}{4 \pi} \sum_{\begin{array}{c}
m, n \in \mathbb{Z} \\
(m, n) \neq(0,0)
\end{array}} \frac{1}{(m+n \tau)^{n+2}} \\
& = \begin{cases}\frac{(n+1) ! \zeta(n+2)}{2 \pi} E_{n+2}, & \text { if } n \text { is even, } \\
0, & \text { if } n \text { is odd. }\end{cases}
\end{aligned}
$$

\section{Appendix B. Graph integrals on $\mathbb{C}$}

In this appendix, we will prove some results for graph integrals on $\mathbb{C}$.

Let $z$ be the linear holomorphic coordinate on $\mathbb{C}, \square=-4 \frac{\partial}{\partial z} \frac{\partial}{\partial \bar{z}}$ be the standard Laplacian operator. The following notations will be used throughout this section

$$
H_{\epsilon}^{L}(z, \bar{z})=\int_{\epsilon}^{L} \frac{d t}{4 \pi t} \mathrm{e}^{-|z|^{2} / 4 t} .
$$

Let $(\Gamma, n)$ be a decorated graph as in Section 3. We will assume that $\Gamma$ is connected without self-loops. We consider the following graph integral on $\mathbb{C}$

$$
\begin{aligned}
W_{(\Gamma, n)}\left(H_{\epsilon}^{L}, \Phi\right) & \equiv \prod_{v \in V(\Gamma)} \int_{\mathbb{C}} d^{2} z_{v}\left(\prod_{e \in E(\Gamma)} \partial_{z_{e}}^{n_{e}} H_{\epsilon}^{L}\left(z_{e}, \bar{z}_{e}\right)\right) \Phi, \\
\text { where } \quad z_{e} & =z_{h(e)}-z_{t(e)}
\end{aligned}
$$

here $\Phi$ is a smooth function on $\mathbb{C}^{|V(\Gamma)|}$ with compact support. In the above integral, we view $H_{\epsilon}^{L}\left(z_{e}, \bar{z}_{e}\right)$ as propagators associated to the edge $e \in E$, and we have only holomorphic derivatives on the propagators.

Proposition B.1. The following limit exists for the above graph integral

$$
\lim _{\epsilon \rightarrow 0} W_{(\Gamma, n)}\left(H_{\epsilon}^{L}, \Phi\right) .
$$


Proof. Let $V=|V(\Gamma)|$ be the number of vertices and $E=|E(\Gamma)|$ be the number of edges. We index the vertices by

$$
v:\{1,2, \ldots, V\} \rightarrow V(\Gamma), \quad V=|V(\Gamma)|
$$

and write $z_{i}$ for $z_{v(i)}$ if there is no confusion. We specify the last vertex by $v$.

$$
v(V)=v_{\bullet} .
$$

Define the incidence matrix $\left\{\rho_{v, e}\right\}_{v \in V(\Gamma), e \in E(\Gamma)}$ by

$$
\rho_{v, e}= \begin{cases}1, & h(e)=v \\ -1, & t(e)=v \\ 0, & \text { otherwise }\end{cases}
$$

and define the $(V-1) \times(V-1)$ matrix $M_{\Gamma}(t)$ as in [6, Section 6-2-3] by

$$
M_{\Gamma}(t)_{i, j}=\sum_{e \in E(G)} \rho_{v(i), e} \frac{1}{t_{e}} \rho_{v(j), e}, \quad 1 \leq i, j \leq V-1,
$$

where $t_{e}$ is a variable introduced for each edge coming from the propagator. Consider the following linear change of variables

$$
\left\{\begin{array}{l}
z_{i}=y_{i}+y_{V}, \quad 1 \leq i \leq V-1 \\
z_{V}=y_{V}
\end{array}\right.
$$

The graph integral can be written as

$$
\begin{aligned}
& W_{(\Gamma, n)}\left(H_{\epsilon}^{L}, \Phi\right) \\
& =\int_{\mathbb{C}} d^{2} y_{V} \int_{\mathbb{C}^{V-1}} \prod_{i=1}^{V-1} d^{2} y_{i} \int_{[\epsilon, L]^{E}} \prod_{e \in E(\Gamma)} \frac{d t_{e}}{4 \pi t_{e}} \prod_{e \in E(\Gamma)}\left(\frac{\sum_{i=1}^{V-1} \rho_{v(i), e} \bar{y}_{i}}{4 t_{e}}\right)^{n_{e}} \\
& \quad \times \exp \left(-\frac{1}{4} \sum_{i, j=1}^{V-1} M_{\Gamma}(t)_{i, j} y_{i} \bar{y}_{j}\right) \Phi .
\end{aligned}
$$


Using integration by parts, we get

$$
\begin{aligned}
W_{(\Gamma, n)}\left(H_{\epsilon}^{L}, \Phi\right)= & \int_{\mathbb{C}} d^{2} y_{V} \int_{\mathbb{C}^{V-1}} \prod_{i=1}^{V-1} d^{2} y_{i} \int_{[\epsilon, L]^{E}} \prod_{e \in E(\Gamma)} \frac{d t_{e}}{4 \pi t_{e}} \\
& \times \exp \left(-\frac{1}{4} \sum_{i, j=1}^{V-1} M_{\Gamma}(t)_{i, j} y_{i} \bar{y}_{j}\right) \\
& \times \prod_{e \in E(\Gamma)}\left(\sum_{j=1}^{V-1} \frac{\sum_{i=1}^{V-1} \rho_{v(i), e} M_{\Gamma}^{-1}(t)_{i, j}}{t_{e}} \frac{\partial}{\partial y_{j}}\right)^{n_{e}} \Phi .
\end{aligned}
$$

By Lemma B.3 below, we see that

$$
\left|\prod_{e \in E(\Gamma)}\left(\sum_{j=1}^{V-1} \frac{\sum_{i=1}^{V-1} \rho_{v(i), e} M_{\Gamma}^{-1}(t)_{i, j}}{t_{e}} \frac{\partial}{\partial y_{j}}\right)^{n_{e}} \Phi\right| \leq C|\tilde{\Phi}|
$$

where $C$ is a constant which doesn't depend on $\left\{t_{e}\right\}$ and $\left\{y_{i}\right\}$, and $\tilde{\Phi}$ is some smooth function with compact support. To prove that $\lim _{\epsilon \rightarrow 0} W_{\Gamma,\left\{n_{e}\right\}}\left(H_{\epsilon}^{L}, \Phi\right)$ exists, we only need to show that

$$
\begin{aligned}
& \lim _{\epsilon \rightarrow 0} \int_{\mathbb{C}^{V-1}} \prod_{i=1}^{V-1} d^{2} y_{i} \int_{[\epsilon, L]^{E}} \prod_{e \in E(\Gamma)} \frac{d t_{e}}{4 \pi t_{e}} \exp \left(-\frac{1}{4} \sum_{i, j=1}^{V-1} M_{\Gamma}(t)_{i, j} y_{i} \bar{y}_{j}\right) \\
& \quad=\lim _{\epsilon \rightarrow 0} \int_{[\epsilon, L]^{E}} \prod_{e \in E(\Gamma)} \frac{d t_{e}}{4 \pi t_{e}} \frac{1}{\operatorname{det} M_{\Gamma}(t)}
\end{aligned}
$$

exists. By Lemma B.1 below, we have

$$
\lim _{\epsilon \rightarrow 0} \int_{[\epsilon, L]^{E}} \prod_{e \in E(\Gamma)} \frac{d t_{e}}{4 \pi t_{e}} \frac{1}{\operatorname{det} M_{\Gamma}(t)}=\lim _{\epsilon \rightarrow 0} \int_{[\epsilon, L]^{E}} \prod_{e \in E(\Gamma)} \frac{d t_{e}}{4 \pi} \frac{1}{\sum_{T \in \operatorname{Tree}(\Gamma)} \prod_{e \notin T} t_{e}}
$$

where Tree $(\Gamma)$ is the set of spanning trees of $\Gamma$. Let $v(1), v(2)$ be two vertices of $\Gamma,\left\{e_{1}, \ldots, e_{k}\right\}$ be the set of edges that connects $v(1), v(2)$. Let $\bar{\Gamma}$ be the graph obtained from $\Gamma$ by collapsing $v(1)$ and $v(2)$ and all the edges $e_{1}, \ldots, e_{k}$ into one single vertex. Then $\bar{\Gamma}$ is also a connected graph without self-loops, with $E(\bar{\Gamma})=E(\Gamma) \backslash\left\{e_{1}, \ldots, e_{k}\right\}$. Obviously, for non-negative $t_{e}$ 's,

$$
\sum_{T \in \operatorname{Tree}(\Gamma)} \prod_{e \notin T} t_{e} \geq\left(\sum_{i=1}^{k} t_{e_{1}} \cdots \hat{t}_{e_{i}} \cdots t_{e_{k}}\right) \sum_{T \in \operatorname{Tree}(\bar{\Gamma})} \prod_{e \notin T} t_{e}
$$


Therefore

$$
\begin{aligned}
& \prod_{e \in E(\Gamma)} \int_{\epsilon}^{L} \frac{d t_{e}}{4 \pi} \frac{1}{\sum_{T \in \operatorname{Tree}(\Gamma)} \prod_{e \notin T} t_{e}} \\
& \leq \prod_{i=1}^{k} \int_{\epsilon}^{L} \frac{d t_{i}}{4 \pi} \frac{1}{\sum_{i=1}^{k} t_{1} \cdots \hat{t}_{i} \cdots t_{k}} \\
& \quad \times \prod_{e \in E(\bar{\Gamma})} \int_{\epsilon}^{L} \frac{d t_{e}}{4 \pi} \frac{1}{\sum_{T \in \operatorname{Tree}(\bar{\Gamma}) \prod_{e \notin T} t_{e}}} \\
& \leq \prod_{i=1}^{k} \int_{\epsilon}^{L} \frac{d t_{i}}{4 \pi} \frac{k}{\prod_{i=1}^{k} t_{i}^{\frac{k-1}{k}}} \\
& \quad \times \prod_{e \in E(\bar{\Gamma})} \int_{\epsilon}^{L} \frac{d t_{e}}{4 \pi} \frac{1}{\sum_{T \in \operatorname{Tree}(\bar{\Gamma})} \prod_{e \notin T} t_{e}} \\
& \leq C(L) \prod_{e \in E(\bar{\Gamma})} \int_{\epsilon}^{L} \frac{d t_{e}}{4 \pi} \frac{1}{\sum_{T \in \operatorname{Tree}(\bar{\Gamma})} \prod_{e \notin T} t_{e}}
\end{aligned}
$$

where $C(L)$ is a constant that depends only on $L$. By successive collapsing of vertices, we see that $\lim _{\epsilon \rightarrow 0} \int_{[\epsilon, L]^{E}} \prod_{e \in E(\Gamma)} \frac{d t_{e}}{4 \pi t_{e}} \frac{1}{\operatorname{det} M_{\Gamma}(t)}$ exists. This proves the lemma.

Definition B.1. A tree $T \subset \Gamma$ is said to be a spanning tree for the connected graph $\Gamma$ if every vertex of $\Gamma$ lies in $T$.

Lemma B.1. The determinant of the $(V-1) \times(V-1)$ matrix $\left\{M_{\Gamma}(t)_{i, j}\right\}_{1 \leq i, j \leq V-1}$ defined by equation (B.1) is given by

$$
\operatorname{det} M_{\Gamma}(t)=\sum_{T \in \operatorname{Tree}(\Gamma)} \prod_{e \in T} \frac{1}{t_{e}}
$$

where Tree $(\Gamma)$ is the set of spanning trees of the graph $\Gamma$.

Proof. See, for example [6, Section 6-2-3].

Definition B.2. Given a connected graph $\Gamma$ and two disjoint subsets of vertices $V_{1}, V_{2} \subset V(\Gamma), V_{1} \cap V_{2}=\emptyset$, we define $\operatorname{Cut}\left(\Gamma ; V_{1}, V_{2}\right)$ to be the set of 
subsets $C \subset E(\Gamma)$ satisfying the following property

(1) The removing of the edges in $C$ from $\Gamma$ divides $\Gamma$ into exactly two connected trees, which we denoted by $\Gamma_{1}(C), \Gamma_{2}(C)$, such that $V_{1} \subset$ $V\left(\Gamma_{1}(C)\right), V_{2} \subset V\left(\Gamma_{2}(C)\right)$.

(2) $C$ does not contain any proper subset satisfying property 1 .

It is easy to see that each cut $C \in \operatorname{Cut}\left(\Gamma ; V_{1}, V_{2}\right)$ is obtained by adding one more edge to some $\{e \in E(\Gamma) \mid e \notin T\}$ where $T$ is some spanning tree of $\Gamma$. Then we have the following result; see [6, Section 6-2-3],

Lemma B.2. The inverse of the matrix $M_{\Gamma}(t)$ is given by

$$
M_{\Gamma}^{-1}(t)_{i, j}=\frac{1}{\mathcal{P}_{\Gamma}(t)} \sum_{C \in \operatorname{Cut}(\Gamma ;\{v(i), v(j)\},\{v \bullet\}} \prod_{e \in C} t_{e}
$$

where

$$
\mathcal{P}_{\Gamma}(t)=\sum_{T \in \operatorname{Tree}(\Gamma)} \prod_{e \notin T} t_{e}=\operatorname{det} M_{\Gamma}(t) \prod_{e \in E(\Gamma)} t_{e}
$$

Proof. Let

$$
A_{i, j}=\frac{1}{\mathcal{P}_{\Gamma}(t)} \sum_{C \in \operatorname{Cut}(\Gamma ;\{v(i), v(j)\},\{v \bullet\})} \prod_{e \in C} t_{e} .
$$

For $1 \leq i \leq V-1$, consider the summation

$$
\begin{aligned}
& \mathcal{P}_{\Gamma}(t) \sum_{j=1}^{V-1} A_{i, j} M_{\Gamma}(t)_{j, i}=\sum_{j=1}^{V-1} M_{\Gamma}(t)_{j, i} \sum_{C \in \operatorname{Cut}\left(\Gamma ;\{v(i), v(j)\},\left\{v_{\bullet}\right\}\right)} \prod_{e \in C} t_{e} \\
& =\sum_{\substack{C \in \operatorname{Cut}\left(\Gamma ;\{v(i)\},\left\{v_{\bullet}\right\}\right) \\
v(i) \in V\left(\Gamma_{1}(C)\right), v_{\bullet} \in V\left(\Gamma_{2}(C)\right)}} \prod_{e \in C} t_{e} \\
& \times \sum_{e^{\prime} \in E(G)} \sum_{\substack{1 \leq j \leq V-1 \\
v(j) \in \Gamma_{1}(C)}} \rho_{v(i), e^{\prime}} \frac{1}{t_{e^{\prime}}} \rho_{v(j), e^{\prime}} \\
& =\sum_{\substack{C \in \operatorname{Cut}\left(\Gamma ;\{v(i)\},\left\{v_{\bullet}\right\}\right) \\
v(i) \in V\left(\Gamma_{1}(C)\right), v \bullet \in V\left(\Gamma_{2}(C)\right)}} \prod_{e \in C} t_{e}
\end{aligned}
$$




$$
\begin{gathered}
\times \sum_{\substack{e^{\prime} \in E(G) \\
l(e)=v(i), r(e) \in V\left(\Gamma_{2}\right) \\
\text { or } r(e)=v(i), l(e) \in V\left(\Gamma_{2}\right)}} \frac{1}{t_{e^{\prime}}} \\
=\sum_{T \in \operatorname{Tree}(\Gamma)} \prod_{e \notin T} t_{e},
\end{gathered}
$$

where in the last step, we use the fact that given $v \neq v_{\bullet}$ and a spanning tree $T$ of $\Gamma$, there is a unique way to remove one edge in $T$, which is attached to $v$, to make a cut that separates $v$ and $v_{\bullet}$. Therefore

$$
\sum_{j=1}^{V-1} A_{i, j} M_{\Gamma}(t)_{j, i}=1, \quad 1 \leq i \leq V-1
$$

Similar combinatorial interpretation leads to

$$
\sum_{k=1}^{V-1} A_{i, k} M_{\Gamma}(t)_{k, j}=0, \quad 1 \leq i, j \leq V_{1}, i \neq j .
$$

We leave the details to the reader. It follows that $A_{i, j}$ is the inverse matrix of $M_{\Gamma}(t)_{i, j}$.

Lemma B.3. The following sum is bounded

$$
\left|\frac{\sum_{i=1}^{V-1} \rho_{v(i), e} M_{\Gamma}^{-1}(t)_{i, j}}{t_{e}}\right| \leq 2, \quad \forall e \in E(G), \quad 1 \leq j \leq V-1 .
$$

Proof.

$$
\begin{aligned}
& \sum_{i=1}^{V-1} \frac{\rho_{v(i), e}}{t_{e}} M_{\Gamma}^{-1}(t)_{i, j} \\
& =\frac{1}{\mathcal{P}_{\Gamma}(t)} \sum_{\substack{C \in \operatorname{Cut}(\Gamma ;\{v(j)\},\{v \bullet\}) \\
v(j) \in V\left(\Gamma_{1}(C)\right), v \bullet \in V\left(\Gamma_{2}(C)\right)}} \prod_{\substack{e^{\prime} \in C \\
t_{e^{\prime}}}} \sum_{\substack{1 \leq i \leq V-1 \\
v(i) \in \Gamma_{1}(C)}} \frac{\rho_{v(i), e}}{t_{e}} \\
& =\frac{1}{\mathcal{P}_{\Gamma}(t)} \sum_{C \in \operatorname{Cut}(\Gamma ;\{v(j), l(e)\},\{v \bullet, r(e)\})} \frac{\prod_{e^{\prime} \in C} t_{e^{\prime}}}{t_{e}}-\frac{1}{\prod_{\Gamma}(t)} \\
& \quad \sum_{C \in \operatorname{Cut}\left(\Gamma ;\{v(j), r(e)\},\left\{v_{\bullet}, l(e)\right\}\right)} \frac{\prod_{e^{\prime} \in C} t_{e^{\prime}}}{t_{e}}
\end{aligned}
$$


Since each cut in the above summation is obtained from removing the edge $e$ from a spanning tree containing $e$, the lemma follows from fact that $\mathcal{P}_{\Gamma}(t)=\sum_{T \in \operatorname{Tree}(\Gamma)} \prod_{e \notin T} t_{e}$ represents the sum of the contributions from all such spanning trees.

Next, we consider another type of graph integral which appears in the proof of Proposition 5.1. Let

$$
P_{\epsilon}^{L}(z, \bar{z})=\int_{\epsilon}^{L} \frac{d t}{4 \pi t}\left(\frac{\bar{z}}{4 t}\right)^{2} \mathrm{e}^{-|z|^{2} / 4 t}
$$

and

$$
U_{\epsilon}(z, \bar{z})=\frac{1}{4 \pi \epsilon}\left(\frac{\bar{z}}{4 \epsilon}\right) \mathrm{e}^{-|z|^{2} / 4 \epsilon} .
$$

Let $(\Gamma, n)$ be a connected decorated graph without self-loops, $V(\Gamma)$ be the set of vertices, $E(\Gamma)$ be the set of edges, $V=|V(\Gamma)|, E=|E(\Gamma)|$. We index the set of vertices as in Proposition B.1 by

$$
v:\{1,2, \ldots, V\} \rightarrow V(\Gamma)
$$

and index the set of edges by

$$
e:\{0,1,2, \ldots, E-1\} \rightarrow E(\Gamma)
$$

such that $e(0), e(1), \ldots, e(k) \in E(\Gamma)$ are all the edges connecting $v(1), v(V)$. We consider the following Feynman graph integral by putting $U_{\epsilon}$ on $e(0)$, putting $P_{\epsilon}^{L}$ to all other edges, and putting a smooth function $\Phi$ on $\mathbb{C}^{|V(\Gamma)|}$ with compact support for the vertices. We would like to compute the following limit of the graph integral

$$
\lim _{\epsilon \rightarrow 0} \prod_{i=1}^{V} \int d^{2} z_{i} \partial_{z_{e(0)}}^{n_{0}} U_{\epsilon}\left(z_{e(0)}, \bar{z}_{e(0)}\right)\left(\prod_{i=1}^{E-1} \partial_{z_{e(i)}}^{n_{i}} P_{\epsilon}^{L}\left(z_{e(i)}, \bar{z}_{e(i)}\right)\right) \Phi,
$$

where we use the notation that

$$
z_{e} \equiv z_{i}-z_{j}, \quad \text { if } h(e)=v(i), t(e)=v(j)
$$


Proposition B.2. The above limit exists and we have the identity

$$
\begin{aligned}
\lim _{\epsilon \rightarrow 0} & \prod_{i=1}^{V} \int d^{2} z_{i} \partial_{z_{e(0)}}^{n_{0}} U_{\epsilon}\left(z_{e(0)}, \bar{z}_{e(0)}\right)\left(\prod_{i=1}^{E-1} \partial_{z_{e(i)}}^{n_{i}} P_{\epsilon}^{L}\left(z_{e(i)}, \bar{z}_{e(i)}\right)\right) \Phi \\
= & \lim _{\epsilon \rightarrow 0} \frac{A\left(n_{0} ; n_{1}, \ldots, n_{k}\right)}{(4 \pi)^{k}} \prod_{i=2}^{V} \int d^{2} z_{i} \partial_{z_{1}}^{n_{0}+1+\sum_{i=1}^{k}\left(n_{i}+2\right)} \\
& \times\left.\left(\left(\prod_{i=k+1}^{E-1} \partial^{n_{i}} P_{\epsilon}^{L}\left(z_{e(i)}, \bar{z}_{e(i)}\right)\right) \Phi\right)\right|_{z_{1}=z_{V}},
\end{aligned}
$$

where the constant $A\left(n_{0}, n_{1}, \ldots, n_{k}\right)$ is a rational number given by

$$
A\left(n_{0} ; n_{1}, \ldots, n_{k}\right)=\int_{0}^{1} \cdots \int_{0}^{1} \prod_{i=1}^{k} d u_{i} \frac{\prod_{i=1}^{k} u_{i}^{n_{i}+1}}{\left(1+\sum_{i=1}^{k} u_{i}\right)^{\sum_{j=0}^{k}\left(n_{j}+2\right)}} .
$$

Proof.

$$
\begin{aligned}
\prod_{i=1}^{V} & \int d^{2} z_{i} \partial^{n_{0}} U_{\epsilon}\left(z_{e(0)}\right)\left(\prod_{i=1}^{E-1} \partial^{n_{i}} H_{\epsilon}^{L}\left(z_{e(i)}\right)\right) \Phi \\
= & \prod_{i=1}^{V} \int d^{2} z_{i} \prod_{i=1}^{E-1} \int_{\epsilon}^{L} d t_{e(i)}\left(\frac{1}{4 \pi \epsilon}\left(\frac{\bar{z}_{e(0)}}{4 \epsilon}\right)^{n_{0}+1}\right) \\
& \times\left(\prod_{i=1}^{E-1} \frac{1}{4 \pi t_{e(i)}}\left(\frac{\bar{z}_{e(i)}}{4 t_{e(i)}}\right)^{n_{i}+2}\right) \mathrm{e}^{-\left(\frac{\left|z_{e(0)}\right|^{2}}{4 \epsilon}+\sum_{j=1}^{E-1} \frac{\left|z_{e(j)}\right|^{2}}{4 t_{e(i)}}\right)} \Phi .
\end{aligned}
$$

We will use the same notations as in the proof of Proposition B.1. The incidence matrix $\left\{\rho_{v, e}\right\}_{v \in V(G), e \in E(G)}$ is defined by

$$
\rho_{v, e}= \begin{cases}1, & h(e)=v \\ -1, & t(e)=v \\ 0, & \text { otherwise }\end{cases}
$$

Without loss of generality, we assume that the orientation of $e(0)$ is such that

$$
\rho_{v(1), e(0)}=1, \rho_{v(V), e(0)}=-1 .
$$


The $(V-1) \times(V-1)$ matrix $M_{\Gamma}(t)$ is defined by

$$
M_{\Gamma}(t)_{i, j}=\sum_{l=0}^{E-1} \rho_{v(i), e(l)} \frac{1}{t_{e(l)}} \rho_{v(j), e(l)}, \quad 1 \leq i, j \leq V-1,
$$

where we use the convention that $t_{e(0)}=\epsilon$. Under the following linear change of variables

$$
\left\{\begin{array}{l}
z_{i}=y_{i}+y_{V}, \quad 1 \leq i \leq V-1 \\
z_{V}=y_{V}
\end{array}\right.
$$

and use integration by parts

$$
\begin{aligned}
\prod_{i=1}^{V} \int d^{2} z_{i} \prod_{i=1}^{E-1} \int_{\epsilon}^{L} d t_{e(i)}\left(\frac{1}{4 \pi \epsilon}\left(\frac{\bar{z}_{e(0)}}{4 \epsilon}\right)^{n_{0}+1}\right)\left(\prod_{i=1}^{E-1} \frac{1}{4 \pi t_{e(i)}}\left(\frac{\bar{z}_{e(i)}}{4 t_{e(i)}}\right)^{n_{i}+2}\right) \\
\times \mathrm{e}^{-\left(\frac{\left|z_{e(0)}\right|^{2}}{4 \epsilon}+\sum_{j=1}^{E-1} \frac{\left|z_{e(j)}\right|^{2}}{4 t_{e(i)}}\right)_{\Phi}} \\
=\int d^{2} y_{V} \prod_{i=1}^{V-1} \int d^{2} y_{i} \prod_{i=1}^{E-1} \int_{\epsilon}^{L} \frac{d t_{e(i)}}{4 \pi t_{e(i)}} \exp \left(-\frac{1}{4} \sum_{i, j=1}^{V-1} y_{i} M_{\Gamma}(t)_{i, j} \bar{y}_{j}\right) \\
\quad \times \frac{1}{4 \pi \epsilon}\left(\sum_{j=1}^{V-1} \frac{\sum_{i=1}^{V-1} \rho_{v(i), e(0)} M_{\Gamma}^{-1}(t)_{i, j}}{\epsilon} \frac{\partial}{\partial y_{j}}\right)^{n_{0}+1} \\
\quad \times \prod_{\alpha=1}^{E-1}\left(\sum_{j=1}^{V-1} \frac{\sum_{i=1}^{V-1} \rho_{v(i), e(\alpha)} M_{\Gamma}^{-1}(t)_{i, j}}{t_{e(\alpha)}} \frac{\partial}{\partial y_{j}}\right)^{n_{\alpha}+2} \Phi .
\end{aligned}
$$

Note that for $0 \leq \alpha \leq k$ and $1 \leq i \leq V-1, \rho_{v(i), e(\alpha)}$ is nonzero only for $\rho_{v(1), e(\alpha)}=1$. Consider the change of variables

$$
\begin{aligned}
t_{e(i)} \rightarrow \epsilon t_{e(i)}, & 1 \leq i \leq k, \\
t_{e(i)} \rightarrow t_{e(i)}, & k+1 \leq i \leq E-1,
\end{aligned}
$$

we get

$$
\begin{gathered}
\int d^{2} y_{V} \prod_{i=1}^{V-1} \int d^{2} y_{i} \prod_{i=1}^{k} \int_{1}^{L / \epsilon} \frac{d t_{e(i)}}{4 \pi t_{e(i)}} \prod_{i=k+1}^{E-1} \int_{\epsilon}^{L} \frac{d t_{e(i)}}{4 \pi t_{e(i)}} \\
\quad \times \exp \left(-\frac{1}{4} \sum_{i, j=1}^{V-1} y_{i} \bar{y}_{j} M_{\Gamma}(\tilde{t})_{i, j}\right)
\end{gathered}
$$




$$
\begin{aligned}
& \times \frac{1}{4 \pi \epsilon}\left(\sum_{j=1}^{V-1} \frac{\sum_{i=1}^{V-1} \rho_{v(i), e(0)} M_{\Gamma}^{-1}(\tilde{t})_{i, j}}{\epsilon} \frac{\partial}{\partial y_{j}}\right)^{n_{0}+1} \prod_{\alpha=1}^{E-1} \\
& \times\left(\sum_{j=1}^{V-1} \frac{\sum_{i=1}^{V-1} \rho_{v(i), e(\alpha)} M_{\Gamma}^{-1}(\tilde{t})_{i, j}}{\tilde{t}_{e(\alpha)}} \frac{\partial}{\partial y_{j}}\right)^{n_{\alpha}+2} \Phi \\
& =\prod_{i=1}^{k} \int_{1}^{L / \epsilon} \frac{d t_{e(i)}}{4 \pi} \prod_{i=k+1}^{E-1} \int_{\epsilon}^{L} \frac{d t_{e(i)}}{4 \pi} F(t ; \epsilon),
\end{aligned}
$$

where $\tilde{t}$ 's are define by

$$
\begin{aligned}
& \tilde{t}_{e(0)}=\epsilon, \quad \text { if } \quad 1 \leq i \leq k, \\
& \tilde{t}_{e(i)}=\epsilon t_{e(i)}, \quad \text { if } \quad k+1 \leq i \leq E-1, \\
& \tilde{t}_{e(i)}=t_{e(i)}, \quad
\end{aligned}
$$

and

$$
\begin{aligned}
F(t ; \epsilon)= & \prod_{i=1}^{V} \int d^{2} y_{i} \frac{1}{\prod_{i=1}^{E-1} t_{e(i)}} \exp \left(-\frac{1}{4} \sum_{i, j=1}^{V-1} y_{i} \bar{y}_{j} M_{\Gamma}(\tilde{t})_{i, j}\right) \\
& \times \frac{1}{4 \pi \epsilon}\left(\sum_{j=1}^{V-1} \frac{\sum_{i=1}^{V-1} \rho_{v(i), e(0)} M_{\Gamma}^{-1}(\tilde{t})_{i, j}}{\epsilon} \frac{\partial}{\partial y_{j}}\right)^{n_{0}+1} \\
& \times \prod_{\alpha=1}^{E-1}\left(\sum_{j=1}^{V-1} \frac{\sum_{i=1}^{V-1} \rho_{v(i), e(\alpha)} M_{\Gamma}^{-1}(\tilde{t})_{i, j}}{\tilde{t}_{e(\alpha)}} \frac{\partial}{\partial y_{j}}\right)^{n_{\alpha}+2} \Phi
\end{aligned}
$$

We first show that $\lim _{\epsilon \rightarrow 0} F(t ; \epsilon)$ exists. Using integration by parts,

$$
\begin{aligned}
F(t ; \epsilon)= & \prod_{i=1}^{V} \int d^{2} y_{i} \frac{1}{\prod_{i=1}^{E-1} t_{e(i)}} \exp \left(-\frac{\left|y_{1}\right|^{2}}{4 \epsilon}\left(1+\sum_{\alpha=1}^{k} \frac{1}{t_{e(\alpha)}}\right)\right) \frac{1}{4 \pi \epsilon} \\
& \times\left(\frac{\bar{y}_{1}}{4 \epsilon}\right)^{n_{0}+1} \prod_{\alpha=1}^{k}\left(\frac{\bar{y}_{1}}{4 \epsilon t_{e(\alpha)}}\right)^{n_{\alpha}+2}
\end{aligned}
$$




$$
\begin{aligned}
& \times \exp \left(-\frac{1}{4} \sum_{i, j=1}^{V-1} y_{i} \bar{y}_{j} \sum_{\beta=k+1}^{E-1} \frac{\rho_{v(i), e(\beta)} \rho_{v(j), e(\beta)}}{t_{e(\beta)}}\right) \\
& \times \prod_{\beta=k+1}^{E-1}\left(\frac{\sum_{i=1}^{V-1} \rho_{v(i), e(\beta)} \bar{y}_{i}}{4 t_{e(\beta)}}\right)^{n_{\alpha}+2} \Phi \\
= & \prod_{i=1}^{V} \int d^{2} y_{i} \frac{1}{\prod_{i=1}^{E-1} t_{e(i)}} \\
\times & \exp \left(-\frac{\left|y_{1}\right|^{2}}{4 \epsilon}\left(1+\sum_{\alpha=1}^{k} \frac{1}{t_{e(\alpha)}}\right)\right) \frac{1}{4 \pi \epsilon}\left(\prod_{\alpha=1}^{k} \frac{1}{t_{e(\alpha)}}\right)^{n_{\alpha}+2} \\
\times & \left(1+\sum_{\alpha=1}^{k} \frac{1}{t_{e(\alpha)}}\right)^{n_{0}+1+\sum_{\alpha=1}^{k}\left(n_{\alpha}+2\right)} \\
& \times\left(\frac{\partial}{\partial y_{1}}\right)^{n_{0}+1+\sum_{\alpha=1}^{k}\left(n_{\alpha}+2\right)}\left(\mathrm{e}^{-\frac{1}{4} \sum_{i, j=1}^{V-1} y_{i} \bar{y}_{j} \sum_{\beta=k+1}^{E-1} \frac{\rho_{v(i), e(\beta)} \rho_{v(j), e(\beta)}}{t_{e(\beta)}}}\right. \\
& \left.\times \prod_{\beta=k+1}^{E-1}\left(\frac{\sum_{i=1}^{V-1} \rho_{v(i), e(\beta)} \bar{y}_{i}}{4 t_{e(\beta)}}\right)^{n_{\alpha}+2} \Phi\right) .
\end{aligned}
$$

Using the property of the heat kernel under the limit $\epsilon \rightarrow 0$, we get

$$
\begin{aligned}
\lim _{\epsilon \rightarrow 0} F(t ; \epsilon)= & \prod_{i=2}^{V} \int d^{2} y_{i} \frac{1}{\prod_{i=1}^{E-1} t_{e(i)}}\left(\prod_{\alpha=1}^{k} \frac{1}{t_{e(\alpha)}}\right)^{n_{\alpha}+2} \\
& \times \frac{1}{\left(1+\sum_{\alpha=1}^{k} \frac{1}{t_{e(\alpha)}}\right)^{\sum_{\alpha=0}^{k}\left(n_{\alpha}+2\right)}} \\
& \times\left(\frac{\partial}{\partial y_{1}}\right)^{n_{0}+1+\sum_{\alpha=1}^{k}\left(n_{\alpha}+2\right)} \\
& \times\left(\mathrm{e}^{-\frac{1}{4} \sum_{i, j=1}^{V-1} y_{i} \bar{y}_{j} \sum_{\beta=k+1}^{E-1} \frac{\rho_{v(i) e(\beta)} \rho_{v(j), e(\beta)}}{t_{e(\beta)}}}\right. \\
& \left.\times \prod_{\beta=k+1}^{E-1}\left(\frac{\sum_{i=1}^{V-1} \rho_{v(i), e(\beta)} \bar{y}_{i}}{4 t_{e(\beta)}}\right)^{n_{\alpha}+2} \Phi\right)\left.\right|_{y_{1}=0}
\end{aligned}
$$




\section{Claim.}

$$
\begin{aligned}
\lim _{\epsilon \rightarrow 0} \prod_{i=1}^{k} \int_{1}^{L / \epsilon} \frac{d t_{e(i)}}{4 \pi} \prod_{i=k+1}^{E-1} \int_{\epsilon}^{L} \frac{d t_{e(i)}}{4 \pi} F(t ; \epsilon) \\
\quad=\prod_{i=1}^{k} \int_{1}^{\infty} \frac{d t_{e(i)}}{4 \pi} \prod_{i=k+1}^{E-1} \int_{0}^{L} \frac{d t_{e(i)}}{4 \pi} \lim _{\epsilon \rightarrow 0} F(t ; \epsilon) .
\end{aligned}
$$

Clearly Proposition B.2 follows from the claim.

To prove the claim, first notice that we have the estimate

$$
0 \leq \frac{M_{\Gamma}^{-1}(t)_{1, j}}{t_{e(\alpha)}} \leq \frac{1}{t_{e(\alpha)}\left(\frac{1}{\epsilon}+\sum_{i=1}^{k} \frac{1}{t_{e(i)}}\right)}
$$

for $1 \leq \alpha \leq k, 1 \leq j \leq V-1$. In fact, by Lemma B.2,

$$
\begin{aligned}
M_{\Gamma}^{-1}(t)_{1, j}= & \frac{\sum_{C \in \operatorname{Cut}\left(\Gamma ;\{v(1), v(j)\},\left\{v_{V}\right\}\right)} \prod_{e \in C} t_{e}}{\sum_{T \in \operatorname{Tree}(\Gamma)} \prod_{e \notin T} t_{e}} \\
\leq & \frac{\sum_{C \in \operatorname{Cut}\left(\Gamma ;\{v(1), v(j)\},\left\{v_{V}\right\}\right)} \prod_{e \in C} t_{e}}{\sum_{\substack{T \in \operatorname{Tree}(\Gamma) \\
e_{i} \in E(T) \text { for some } 0 \leq i \leq k}} \prod_{e \notin T} t_{e}} \leq \frac{1}{\left(\frac{1}{\epsilon}+\sum_{i=1}^{k} \frac{1}{t_{e(i)}}\right)}
\end{aligned}
$$

For $0 \leq \alpha \leq E-1,1 \leq j \leq V-1, \frac{\sum_{i=1}^{V-1} \rho_{v(i), e(\alpha)} M_{\Gamma}^{-1}(t)_{i, j}}{t_{e(\alpha)}}$ is bounded by a constant by Lemma B.3. It follows that

$$
\begin{aligned}
|F(t ; \epsilon)| \leq & \prod_{i=1}^{V} \int d^{2} y_{i} \frac{1}{\prod_{i=1}^{E-1} t_{e(i)}} \exp \left(-\frac{1}{4} \sum_{i, j=1}^{V-1} y_{i} \bar{y}_{j} M_{\Gamma}(\tilde{t})_{i, j}\right) \\
& \times \mid \frac{1}{4 \pi \epsilon}\left(\sum_{j=1}^{V-1} \frac{\sum_{i=1}^{V-1} \rho_{v(i), e(0)} M_{\Gamma}^{-1}(\tilde{t})_{i, j}}{\epsilon} \frac{\partial}{\partial y_{j}}\right)^{n_{0}+1} \\
& \times \prod_{\alpha=1}^{E-1}\left(\sum_{j=1}^{V-1} \frac{\sum_{i=1}^{V-1} \rho_{v(i), e(\alpha)} M_{\Gamma}^{-1}(\tilde{t})_{i, j}}{\tilde{t}_{e(\alpha)}} \frac{\partial}{\partial y_{j}}\right)^{n_{\alpha}+2} \Phi \mid
\end{aligned}
$$




$$
\begin{aligned}
\leq & \prod_{i=1}^{V} \int d^{2} y_{i} \frac{1}{\prod_{i=1}^{E-1} t_{e(i)}} \exp \left(-\frac{1}{4} \sum_{i, j=1}^{V-1} y_{i} \bar{y}_{j} M_{\Gamma}(\tilde{t})_{i, j}\right) \frac{1}{4 \pi \epsilon} \\
& \times \prod_{1 \leq \alpha \leq k}\left(\frac{1}{t_{e(\alpha)}\left(1+\sum_{i=1}^{k} \frac{1}{t_{e(i)}}\right)}\right)^{n_{\alpha}+2} \tilde{\Phi},
\end{aligned}
$$

where $\tilde{\Phi}$ is some non-negative smooth function on $\mathbb{C}^{V}$ with compact support. Integrating over $y_{i}$ 's we get

$$
\begin{aligned}
|F(t ; \epsilon)| \leq & C \frac{1}{\prod_{i=1}^{E-1} t_{e(i)}} \frac{1}{\epsilon \operatorname{det} M_{\Gamma}(\tilde{t})} \prod_{1 \leq \alpha \leq k}\left(\frac{1}{t_{e(\alpha)}\left(1+\sum_{i=1}^{k} \frac{1}{t_{e(i)}}\right)}\right)^{n_{\alpha}+2} \\
= & C \frac{\epsilon^{k}}{\mathcal{P}_{\Gamma}\left(\epsilon, \epsilon t_{e(1)}, \ldots, \epsilon t_{e(k)}, t_{e_{k+1}}, \ldots, t_{e(E-1)}\right)} \\
& \times \prod_{1 \leq \alpha \leq k}\left(\frac{1}{t_{e(\alpha)}\left(1+\sum_{i=1}^{k} \frac{1}{t_{e(i)}}\right)}\right)^{n_{\alpha}+2} \\
\leq & C \frac{1}{\mathcal{P}_{\bar{\Gamma}}\left(t_{e_{k+1}}, \ldots, t_{e(E-1)}\right) \prod_{\alpha=1}^{k} t_{e(\alpha)}} \prod_{\alpha=1}^{k} \frac{1}{t_{e(\alpha)}^{n_{\alpha}+2}},
\end{aligned}
$$

where $C$ is a constant that only depends on $\tilde{\Phi}, \bar{\Gamma}$ is the graph obtained by collapsing the vertices $v(1), v(V)$ and all $e(0), e(1), \ldots, e(k)$, and $\mathcal{P}_{\Gamma}$ is defined in Lemma B.2. Here we have used the simple combinatorial fact that

$$
\begin{aligned}
& \mathcal{P}_{\Gamma}\left(\epsilon, \epsilon t_{e(1)}, \ldots, \epsilon t_{e(k)}, t_{e_{k+1}}, \ldots, t_{e(E-1)}\right) \\
& \quad \geq \epsilon^{k}\left(\prod_{\alpha=1}^{k} t_{e(\alpha)}\right)\left(1+\sum_{\alpha=1}^{k} \frac{1}{t_{e(\alpha)}}\right) \mathcal{P}_{\bar{\Gamma}}\left(t_{e_{k+1}}, \ldots, t_{e(E-1)}\right) .
\end{aligned}
$$

Since $\bar{\Gamma}$ has no self-loops,

$$
\prod_{i=1}^{k} \int_{1}^{\infty} \frac{d t_{e(i)}}{4 \pi} \prod_{\alpha=1}^{k} \frac{1}{t_{e(\alpha)}^{n_{\alpha}+3}} \prod_{i=k+1}^{E-1} \int_{0}^{L} \frac{d t_{e(i)}}{4 \pi} \frac{1}{\mathcal{P}_{\bar{\Gamma}}\left(t_{e_{k+1}}, \ldots, t_{e(E-1)}\right)}<\infty .
$$

Now the claim follows from dominated convergence theorem. 


\section{References}

[1] M. Aganagic, V. Bouchard and A. Klemm, Topological strings and (almost) modular forms, Comm. Math. Phys. 277(3) (2008), 771-819, doi 10.1007/s00220-007-0383-3. MR2365453 (2008m:81174).

[2] M. Bershadsky, S. Cecotti, H. Ooguri and C. Vafa, Kodaira-spencer theory of gravity and exact results for quantum string amplitudes, Comm. Math. Phys. 165(2) (1994), 311-427. MR1301851 (95f:32029).

[3] K. Costello and S. Li, Quantum BCOV theory on Calabi-Yau manifolds and the higher genus B-model, 2012, arXiv:math.QA/1201.4501.

[4] S. Li, BCOV theory on the elliptic curve and higher genus mirror symmetry, 2011, arXiv:math.QA/1112.4063.

[5] R. Dijkgraaf, Mirror symmetry and elliptic curves, in: The moduli space of curves, (Texel Island, 1994), Progr. Math., 129, Birkhäuser Boston, Boston, MA, 1995, 149-163, MR1363055 (96m:14072).

[6] C. Itzykson and J. B. Zuber, Quantum field theory, McGraw-Hill International Book Co., New York, Int. Ser. Pure Appl. Phys., 1980.

[7] M. Kaneko and D. Zagier, A generalized Jacobi Theta function and quasimodular forms, in: The moduli space of curves, (Texel Island, 1994), Progr. Math., 129, Birkhäuser Boston, Boston, MA, 1995, 165-172, MR1363056 (96m:11030).

[8] S. Li, Calabi-Yau geometry and higher genus mirror symmetry. PhD thesis, Harvard University, 2011, 174 pp.

[9] J.-P. Serre, A course in arithmetic, Grad. Texts in Math. 7, SpringerVerlag, New York, 1973. Translated from the French; MR0344216 (49 \#8956).

Mathematics Department

NORTHWESTERN UNIVERSITY

EVANSTON, IL 60201

USA

E-mail address: sili@math.northwestern.edu

Received October 18, 2011 
